\title{
APPLICATIONS OF EXTREMAL LENGTH TO CLASSIFICATION OF RIEMANN SURFACES
}

\author{
TATSUO FUJI'I'E
}

\section{Introduction}

Let $D$ be a subregion of a Riemann surface $F$, whose relative boundary consists of at most countable number of analytic curves which do not cluster in $F$. For a regular exhaustion $\left\{F_{n}\right\}$ of $F$, we put $D_{n}=D \cap\left(F-F_{n}\right)$, and define the extremal radius $R\left(P, \partial D_{n}\right)$ of the relative boundary $\partial D_{n}$ of $D_{n}$, measured at a point $P\left(\in F_{0}\right)$ of $F$ with respect to the connected component of $F-D_{n}$ which contains $P$. Let $K(|z| \leqq r)$ be a disk centered at $P$ and contained in a parametric disk of $P$. And let $\lambda_{n, r}$ be the extremal length of the family of rectifiable curves which join $\partial K$ and $\partial D_{n}$. Then, the extremal radius $R\left(P, \partial D_{n}\right)$ is defined as follows [2];

$$
R\left(P, \partial D_{n}\right)=\lim _{r \rightarrow 0} r e^{2 \pi \lambda_{n, r}}
$$

And we put

$$
R\left(P, B_{D}\right)=\lim _{n \rightarrow \infty} R\left(P, \partial D_{n}\right) .
$$

Taking $F$ as $D$, we define the extremal radius $R(P, B)=\lim _{r \rightarrow 0} r e^{2 \pi \mu_{r}}$ of the ideal boundary $B$ of $F$, where $\mu_{r}$ is the extremal length of the family of locally rectifiable curves which start from $\partial K$ and tend to the ideal boundary $B$ of $F$.

In $\S 1$ we show that it is necessary and sufficient for $F$ not to belong to the class $O_{H D}$ that there exists a subregion $D$ of $F$ for which $\infty>R\left(P, B_{D}\right)>$ $R(P, B)$.

In $\S 2$ we consider a subregion $W$ in place of the Riemann surface $F$. The corresponding extremal radii are denoted by $R^{\prime}\left(P, B_{D}\right)$ and $R^{\prime}(P, B)$.

Then, the existence of a subregion $D$ of $W$ such that $\infty>R^{\prime}\left(P, B_{D}\right)>$ $R^{\prime}(P, B)$ is necessary and sufficient for $W$ not to belong to the class $N O_{H D}{ }^{\prime \prime}$.

Received November 28, 1963.

1) $N O_{H D}\left(S O_{H D}\right)$ denotes the class of subregions on which there are no non-constant harmonic functions with finite Dirichlet integral whose normal derivatives are zero (which are zero, respectively) on the relative boundary. 
And we consider the extremal radius $R^{\prime}(P, \partial W)$ of the relative boundary $\partial W$ of $W$ and the extremal radius $R^{\prime}(P, \partial W \cup B)$ of the union of $\partial W$ and the ideal boundary $B$ of $W$. Then, $W$ does not belong to the class $S O_{H D}$ if and only if $R^{\prime}(P, \partial W)>R^{\prime}(P, \partial W \cup B)$.

Some applications of the theorems are also showed in this section.

\section{$\S 1$. A criterion for the class $O_{H D}$ of Riemann surfaces}

In order to evaluate the extremal lengh $\lambda_{n, r}$, we consider the following harmonic function $U_{n, i}$ in $\left\{\left(F-D_{n}\right) \cap F_{n+i}\right\}-P^{2)}$

$$
U_{n, i}: \begin{cases}U_{n, i}=-\log |z|+u_{n, i} & \text { in a neighborhood of } P \text {, where } u_{n, i} \text { is harmonic } \\ U_{n, i}=0 & \text { on } \partial D_{n} \cap F_{n+i} \\ \frac{U_{n, i}}{\partial n}=0 & \text { on } \partial F_{n+i} \cap\left(F-D_{n}\right) .\end{cases}
$$

Since the sequence $\left\{U_{n, i}\right\}_{i}$ converges in the sense of Dirichlet norm ${ }^{3}$, it converges uniformly to a limit function $U_{n}$ on every compact set in $F-D_{n}$. The extremal length $\lambda_{n, i, r}$ of the family of curves which join $\partial K$ and $\partial D_{n} \cap F_{n+i}$ decreases monotonely when $i$ increases. So,

$$
\lambda_{n, i, r} \geqq \lim _{i \rightarrow \infty} \lambda_{n, i, r} \geqq \lambda_{n, r} .
$$

But, denoting by $U_{n, i, r}$ a harmonic function in $\left(F-D_{n}\right) \cap F_{n+i}-K$ which is zero on $\partial D_{n} \cap F_{n+i}$, equals $-\log r$ on $\partial K$, and whose normal derivative is zero on $\partial F_{n+i} \cap\left(F-D_{n}\right)$,

$$
\lambda_{n, i, r}=\frac{(\log r)^{2}}{D\left(U_{n, i, r}\right)}
$$

and

$$
\lambda_{n, r} \geqq \frac{(\log r)^{2}}{D\left(U_{n, r}\right)}=\lim _{i \rightarrow \infty} \frac{(\log r)^{2}}{D\left(U_{n, i, r}\right)}
$$

where $U_{n, r}=\lim _{i \rightarrow \infty} U_{n, i, r}$.

Hence

$$
\lambda_{n, r}=\lim _{i \rightarrow \infty} \lambda_{n, i, r}=\frac{(\log r)^{2}}{D\left(U_{n, r}\right)}
$$

2) When $\left\{\left(F-D_{n}\right) \cap F_{n+i}\right\}$ is not connected, we take a connected component which contains $P$.

3) $\lim _{i \rightarrow \infty} D\left(U_{n, i+p}-U_{n, i}\right)=0$. (cf. Strebel [2] p. 8). 
While,

$$
2 \pi \frac{(\log r)^{2}}{D\left(U_{n, r}\right)}=-\log r+u_{n}(0)+o(1),
$$

where $u_{n}=\lim _{i \rightarrow \infty} u_{n, i}$. We conclude that

$$
R\left(P, \partial D_{n}\right)=e^{u_{n}(0)} .
$$

And by our definition $R\left(P, B_{D}\right)=\lim _{n \rightarrow \infty} R\left(P, \partial D_{n}\right)$.

Using this extremal radius we get the following theorem.

TheOREM 1. A Riemann surface $F$ does not belong to the class $O_{H D}$ if and only if there exists a subregion $D$ of $F$ such that

$$
\infty>R\left(P, B_{D}\right)>R(P, B) .
$$

For the proof of the theorem, we prove the following lemma.

Lemma. If the double $\hat{D}$ of $D$ is not of the class $O_{G}$, the limit function $U_{B_{D}}=\lim _{n \rightarrow \infty} U_{n}^{5)}$ is not constantly infinite.

Proof of the lemma. By adding to $D$ a suitable relatively compact subregion $\Delta$ which contains $P$, we build up a (connected) subregion $D^{\prime}=D \cup \Delta$ whose double $\hat{D}^{\prime}$ is not of the class $O_{G}$. The extremal length of the family of curves in $\hat{D}^{\prime}$ which start from $\partial K \cup(\partial K)^{\sim}\left((\partial K)^{\sim}\right.$ is a symmetric image of $\partial K$ in $\left.\hat{D}^{\prime}-D^{\prime}\right)$ and tend to the ideal boundary of $\hat{D}^{\prime}$ is finite because $\hat{D}^{\prime} \notin O_{G}$. Then, by the method of symmetrization [3], the extremal length $\lambda_{A}^{\prime}$, with respect to $D^{\prime}$, of the family $A$ of curves in $D^{\prime}$ which start from $\partial K$ and tend to the ideal boundary of $D^{\prime}$ is finite. Now, we consider a family $B$ of curves in $F$, each curve of which contains a curve connecting $\partial K$ and $\partial D_{n}$ for all $n$. Then the family $B$ contains the family $A$, so the extremal length $\lambda_{B}$ of $B$ with respect to $F$ is smaller than the extremal length $\lambda_{A}$ of $A$ with respect to $F$. And,

$$
\lambda_{B} \leqq \lambda_{A}=\lambda^{\prime}{ }_{A}<\infty .
$$

But,

$$
\frac{(\log r)^{2}}{D\left(U_{n, r}\right)}=\lambda_{n, r} \leqq \lambda_{B}<\infty
$$

4) About these calculation, confer Strebel's paper ([2] p. 13).

5) According to Strebel, we call $U_{B D}$ "Strömungspotential". 
and

$$
\lim _{n \rightarrow \infty} \frac{(\log r)^{2}}{D\left(U_{n, r}\right)}=\lim _{n \rightarrow \infty} \lambda_{n, r} \leqq \lambda_{B}<\infty .
$$

So, $U_{r}=\lim _{n \rightarrow \infty} U_{n, r}$ is not a constant, and from

$$
2 \pi \frac{(\log r)^{2}}{D\left(U_{n, r}\right)}=-\log r+u_{n}(0)+o(1)
$$

$\lim _{n \rightarrow \infty} u_{n}(0)$ is finite.

Therefore, for a sufficiently large number $L$,

$$
U_{B_{D}}=\lim _{n \rightarrow \infty} U_{n} \leqq \lim _{n \rightarrow \infty} U_{n, r}+L
$$

in $F-K$, and this shows that $U_{B n}$ is not constantly infinite in $F$.

Proof of the Theorem. If $F$ is not of the class $O_{H D}$, there are two disjoint subregions $D$ and $S$ neither of which is of the class $S O_{H D}$. And we suppose that the point $P$ and its parametric disk $K$ are contained in $S$.

For a regular exhaustion $\left\{F_{n}\right\}$ of $F$, we construct a harmonic function $v_{n}$ in $F_{n} \cap S^{6)}$ such that

$$
v_{n}: \begin{cases}v_{n} & \text { has a positive logarithmic pole at } P \\ v_{n}=0 & \text { on } \partial S \cap F_{n} \\ \frac{\partial v_{n}}{\partial n}=0 & \text { on } \partial F_{n} \cap S .\end{cases}
$$

$v_{n}$ tends to a limit function $v=\lim _{n \rightarrow \infty} v_{n}$ as above, and $v$ is not constant because $v$ has a logarithmic pole at $P$ and $v=0$ on $\partial S$. Let $g$ be Green's function of $S$ with a pole at $P$. Then, by Kuramochi's theorem (Kuramochi [1] p. 135),

$$
v>g
$$

because $S \notin S O_{H D}$.

On the other hand, since $D \notin S O_{H D}$, the double $\hat{D}$ does not belong to $O_{G}$. So, by the lemma, there exists a non-constant limit function $U_{B D}$ of $U_{n}$. Now, we prove in the following that the inequality

$$
U_{B D}-G \geqq v-g
$$

holds in $S$, where $G$ is Green's function of $F$. Let $G_{n, i}$ be Green's function of

6) We take a connected component of $F_{n} \cap S$ which contains $P$. 
$\left(F-D_{n}\right) \cap F_{n+i}$ with a pole at the point $P$, and $g_{n+i}$ be Green's function of $F_{n+i} \cap S$ with a pole at $P$. We prove the above inequality in three steps.

1) Since $U_{n, i}-v_{n+i}$ is harmonic in $F_{n+i} \cap S$ and

$$
\begin{cases}U_{n, i}-v_{n+i} \geqq 0 & \text { on } \partial S \cap F_{n+i} \\ \frac{\partial\left(U_{n, i}-v_{n+i}\right)}{\partial n}=0 & \text { on } \partial F_{n+i} \cap S\end{cases}
$$

we have $U_{n, i}-v_{n+i} \geqq 0$ in $F_{n+i} \cap S$, especially on $\partial F_{n+i} \cap S$.

2) Since $v_{n+i}=g_{n+i}=0$ on $\partial S \cap F_{n+i}$,

$$
U_{n, i}-G_{n, i}-\left(v_{n+i}-g_{n+i}\right)= \begin{cases}U_{n, i}-G_{n, i} \geqq 0 & \text { on } \partial S \cap F_{n+i} \\ U_{n, i}-v_{n+i} \geqq 0 & \text { on } \partial F_{n+i} \cap S .\end{cases}
$$

So, we have

$$
U_{n, i}-G_{n, i}-\left(v_{n+i}-g_{n+i}\right) \geqq 0 \quad \text { on } \partial\left(\dot{S} \cap F_{n+i}\right) .
$$

Hence,

$$
U_{n, i}-G_{n, i}-\left(v_{n+i}-g_{n+i}\right) \geqq 0 \quad \text { in } S \cap F_{n+i} .
$$

3) Here, let $i$ tend to $\infty$, then

$$
U_{n}-G_{n} \geqq v-g \quad \text { in } S .
$$

Since this inequality is valid for all $n$, we have

$$
U_{B D}-G \geqq v-g>0 \quad \text { in } S \text {. }
$$

And

$$
U_{B D}-G \geqq 0 \quad \text { in } F
$$

from the start. Consequently,

$$
U_{B_{D}}-G>0 \text { in } F .
$$

But, if we put $u=\lim _{n \rightarrow \infty} \lim _{i \rightarrow \infty} u_{n, i}$, then $U_{B_{D}}=-\log r+u$ in the neighborhood of $P$, and $R\left(P, B_{D}\right)=e^{u(0)}$. And

$$
R(P, B)=e^{h(0)},
$$

where $G=-\log r+h$ in the neighborhood of $P$. Therefore,

$$
R\left(P, B_{D}\right)>R(P, B) \text {. }
$$

And $R\left(P, B_{D}\right)<\infty$ from the lemma. 
Conversely, we suppose that there exists a subregion $D$ such that $\infty>$ $R\left(P, B_{D}\right)>R(P, B)$. Then, $U_{B_{D}}-G$ is a non-constant harmonic function with finite Dirichlet integral. And $F$ does not belong to $O_{H D}$.

Namely, if $U_{B D}-G$ is a constant, $D_{F-|z|<r}\left(U_{B D}\right)=D_{F-|z|<r}(G)$. And since

$$
D\left(U_{r}-U_{B_{D}}\right)=o(1) \text { and } D\left(G-G_{r}\right)=o(1)
$$

we have $D\left(U_{r}\right)=D\left(G_{r}\right)+o(1)$. Here, $G_{r}$ is a harmonic function in $F-(|z| \leqq r)$ with boundary values $\log 1 / r$ on $|z|=r$ and zero on the ideal boundary of $F$. While, from $R\left(P, B_{D}\right)>R(P, B)$, we have

$$
\lim _{n \rightarrow \infty} \lambda_{n, r}-\mu_{r}>\frac{1}{2 \pi} \log \left(\frac{d}{r e^{2 \pi \mu_{r}}}+1\right)
$$

with a positive constant $d$, in $F-(|z| \leqq r)$ for sufficiently small $r$. This is a contradiction, because

$$
\lim _{n \rightarrow \infty} \lambda_{n, r}-\mu_{r}=\frac{(\log r)^{2}}{D\left(U_{r}\right)}-\frac{(\log r)^{2}}{D\left(G_{r}\right)}=(\log r)^{2} \frac{D\left(G_{r}\right)-D\left(U_{r}\right)}{D\left(U_{r}\right) D\left(G_{r}\right)}
$$

and $D\left(U_{r}\right) D\left(G_{r}\right) \sim(\log r)^{2}$.

Remark. In the proof of Theorem 1, it is also proved that if there exist two such subregions $D$ and $S$ on a Riemann surface $F$ that $\hat{D}$ is not of the class $O_{G}$ and $S$ is not of the class $S O_{H D}$, then, the Riemann surface $F$ is not of the class $O_{H D}$.

\section{§ 2. Subregion}

In this section we consider a subregion $W$, and put $\bar{W}=W+\partial W$. We choose a sequence $\left\{W_{n}\right\}$ (exhaustion of $W$ ) of relatively compact subregions $W_{n}$ such that the relative boundary $\partial W_{n}$ of $W_{n}$ consists of closed curves in $W$, cross-cuts ending at $\partial W$ and parts of $\partial W$, and such that the intersection of the closures $\left\{\overline{W-W_{n}}\right\}$ of $\left\{W-W_{n}\right\}$ in $W$ is empty. Then, the sequence $\left\{W-W_{n}\right\}$ defines the ideal boundary $B$ of $W$. For a relatively non-compact subregion $D$ of $\bar{W}$ we put $D_{n}=D \cap\left(W-W_{n}\right)$. Let $\lambda_{n, i, r}$ be the extremal length of the family of curves in $W_{n+i}-D_{n}$ which join $\partial K$ and $\partial D_{n} \cap \bar{W}_{n+i}$. Then $\lambda_{n, r}=\lim _{i \rightarrow \infty} \lambda_{n, i, r}$ is the extremal length of the family of curves in $F-D_{n}$ which join $\partial K$ and $\partial D_{n}$ and we put $\lambda_{r}=\lim _{n \rightarrow \infty} \lambda_{n, r}$. And let $\mu_{r}$ be the extremal distance between $\partial K$ and the ideal boundary $B$. By putting $R(P, B)=\lim _{r \rightarrow 0} r e^{2 \pi \mu_{r}}$ and 
$R\left(P, B_{D}\right)=\lim _{r \rightarrow 0} r e^{2 \pi \lambda_{r}}$, we have the following theorem.

THEOREM 2. $W$ does not belong to $N O_{H D}$ if and only if there exists a sub. region $D$ of $W$ such that

$$
\infty>R\left(P, B_{D}\right)>R(P, B) .
$$

Proof. If $W$ is not of the class $N O_{H D}$, the double $\hat{W}$ of $W$ is not of the class $O_{H D}$ and we can find two disjoint subregions $D^{\prime}$ and $F^{\prime}$ each of which is symmetric and not of the class $S O_{H D}$. We write $D^{\prime}=D \cup \widetilde{D}$ and $F^{\prime}=F \cup \widetilde{F}$.

As in the proof of Theorem 1, we construct the "Strömungspotential" $U_{B_{D}}$ with respect to $D^{\prime}$ and a point $P$ in $W$ and the "Strömungspotential" $U_{B_{D}}(\widetilde{P})$ with respect to $D^{\prime}$ and the symmetric point $\widetilde{P}$ of $P$. Let $G^{\prime}(P)$ and $G^{\prime}(\widetilde{P})$ be Green's functions of $W$ with the pole at $P$ and the symmetric point $\widetilde{P}$ of $P$, respectively. And we put

$$
\begin{aligned}
U_{B_{D}} & =\frac{1}{2}\left(U_{B_{D}}^{\prime}(P)+\widetilde{U}_{B_{D}}^{\prime}(\widetilde{P})\right), \\
G & =\frac{1}{2}\left(G^{\prime}(P)+\widetilde{G}^{\prime}(\widetilde{P})\right) .
\end{aligned}
$$

Then the normal derivatives of them along $\partial W$ are zero. And since

$$
U_{B_{D}}^{\prime}(P)>G^{\prime}(P) \text { and } U_{B_{D}}^{\prime}(\widetilde{P})>G^{\prime}(\widetilde{P}),
$$

we have

$$
U_{B_{\nu}}>G
$$

Hence, as in Theorem 1 we have

$$
\infty>R\left(P, B_{D}\right)>R(P, B) .
$$

Converse is also true. If there exists a subregion $D$ of $W$ for which

$$
\infty>R\left(P, B_{D}\right)>R(P, B),
$$

we find as the proof of Theorem 1 that $U_{B_{D}}-G$ is a non-constant harmonic function with finite Dirichlet integral whose normal derivative on $\partial W$ is zero. Hence, $W$ is not of the class $N O_{H D}$.

Denoting by $R(P, \partial W)$ and $R(P, B(W))$ the extremal radii, measured at a point $P$, of the relative boundary $\partial W$ of $W$ and the whole boundary $B(W)=\partial W+$ (ideal boundary) of $W$, respectively, we have the following theorem as a direct consequence of Kuramochi's theorem. 
Theorem 3. A subregion $W$ is not of the class $\mathrm{SO}_{\mathrm{HD}}$ if and only if

$$
R(P, \partial W)>R(P, B(W)) .
$$

As applications of Theorems 2 and 3 to the plane regions, we consider a closed set $E$ on the unit circle $|z|=1$. We set $W=|z|<1$ and $\partial W=(|z|=1)-E$. Then, $W$ is of the class $N O_{H D}$ if and only if capacity of $E$ is zero. $E$ is of the class $N_{D}$ if and only if $R(P,|z|=1)=R(P, \partial W)$ because if $E$ is of the class $N_{D} W$ is of the class $S O_{H D}$ and vice versa.

\section{REFERENCES}

[1] Z. Kuramochi. Singular points of Riemann surfaces, J. Fac. Sci. Hokkaido Univ. Ser. I vol. XVI. (1962) pp. 80-148.

[2] K. Strebel. Die Extremale Distanz zweier Enden einer Riemannschen Fläche, Ann. Acad. Sci. Fennicae., A.I. 179 (1955) pp. 1-21.

[3] V. Wolontis. Properties of conformal invariants, Amer. J. Math. LXXIV. (1952) pp. 587-606. 